\title{
Suitability of UML4ODP for an Engineering Specification of a Distributed Environment for Teaching Embedded Systems
}

\author{
Mohamed Mhamdi, Technologist Professor ${ }^{1}$, Hamadou Saliah-Hassane, Senior IEEE Member ${ }^{2}$ \\ Rafik Braham, Professor ${ }^{3}$, Wahid Bannour, Technologist Lecturer ${ }^{4}$ \\ ${ }^{1}$ ISET, Higher Institute of Technology of Sousse, Tunisia, m_mhamdi@yahoo.fr \\ ${ }^{2}$ TELUQ, University of Quebec, Canada, hamadou.saliah-hassane@teluq.ca \\ ${ }^{3}$ PRINCE Research Unit of Sousse, Tunisia, rafik.braham@ensi.rnu.tn \\ ${ }^{4}$ ISET, Higher Institute of Technology of Mahdia, Tunisia, bannourwahid@ gmail.com
}

\begin{abstract}
In this paper, we will contribute to an engineering specification of a distributed environment that will help to generate remote Lab work supports for teaching embedded systems. Our main objective is to show the appropriateness of adopting the ODPRM (Open Distributed Processing - Reference Model) model proposed by the ISO (International Organization for Standardization) to express the specification of our environment from an engineering point of view. By choosing the UML4ODP language that comprises all the ODP-RM concepts to express the specification of such point of view we will try to specify our environment as a distributed system composed of a set of objects and that support the necessary mechanisms and functions for their distributed interactions. Software architecture styles, such as SOA, $M V C$ and $N$-tier, are considered in our engineering specification, since they are closely related to the distribution strategy. Keywords- ODP-RM, UML4ODP, Distributed Environment, Remote lab work, Online laboratory.
\end{abstract}

Digital Object Identifier (DOI):

http://dx.doi.org/10.18687/LACCEI2016.1.1.383

ISBN: 978-0-9822896-9-3

ISSN: 2414-6390

$14^{\text {th }}$ LACCEI International Multi-Conference for Engineering, Education, and Technology: "Engineering Innovations for Global Sustainability", 20-22 July 2016, San José, Costa Rica. 


\title{
Suitability of UML4ODP for an Engineering Specification of a Distributed Environment for Teaching Embedded Systems
}

\author{
Mohamed Mhamdi, Technologist Professor ${ }^{1}$, Hamadou Saliah-Hassane, Senior IEEE Member ${ }^{2}$ \\ Rafik Braham, Professor ${ }^{3}$, Wahid Bannour, Technologist Lecturer ${ }^{4}$ \\ ${ }^{1}$ ISET, Higher Institute of Technology of Sousse, Tunisia, m_mhamdi@yahoo.fr \\ ${ }^{2}$ TELUQ, University of Quebec, Canada, hamadou.saliah-hassane@teluq.ca \\ ${ }^{3}$ PRINCE Research Unit of Sousse, Tunisia, rafik.braham@ensi.rnu.tn \\ ${ }^{4}$ ISET, Higher Institute of Technology of Mahdia, Tunisia, bannourwahid@gmail.com
}

\begin{abstract}
In this paper, we will contribute to an engineering specification of a distributed environment that will help to generate remote Lab work supports for teaching embedded systems. Our main objective is to show the appropriateness of adopting the ODPRM (Open Distributed Processing - Reference Model) model proposed by the ISO (International Organization for Standardization) to express the specification of our environment from an engineering point of view.

By choosing the UML4ODP language that comprises all the ODP-RM concepts to express the specification of such point of view we will try to specify our environment as a distributed system composed of a set of objects and that support the necessary mechanisms and functions for their distributed interactions.
\end{abstract}

Software architecture styles, such as SOA, MVC and N-tier, are considered in our engineering specification, since they are closely related to the distribution strategy.

Keywords-- ODP-RM, UML4ODP, Distributed Environment, Remote lab work, Online laboratory.

\section{INTRODUCTION}

This work presents original and innovative ways of considering research axes on Online Laboratories based on the acquired experience within the framework of two projects carried on at LICEF Research Center in Montreal: LORNET and EduSource projects.

The main goal of the EduSource project was to create a repository of interoperable networked learning objects. In the framework of LORNET project, laboratory components as learning objects stored in repositories was presented in [1].

One of LORNET project's goal was to define a generic concept of an online laboratory allowing students to realize practical works remotely and as well as in collaboration. This generic architecture is based on the specification defined by most known e-learning standards. It is also based on the use of ontological model to describe the controlled devices of the embedded automated systems.

We recall that the main objective of this paper is to perform, based in the ODP- RM (Open Distributed Processing

Digital Object Identifier (DOI): http://dx.doi.org/10.18687/LACCEI2016.1.1.383 ISBN: 978-0-9822896-9-3

ISSN: $2414-6390$

14 ${ }^{\text {th }}$ LACCEI International Multi-Conference for Engineering, Education, and Technology: "Engineering Innovations for Global Sustainability", 20-22 July 2016, San José, Costa Rica.
- Reference Model) [2], an engineering specification of a distributed environment that will help to generate distance lab work or Remote lab work supports for teaching embedded systems. We called this environment "a Tele-LabWork System Environment Generator for Teaching Embedded System" or "TeleLWS_EG", for short.

Three Software architecture styles (N-tier, MVC, SOA) related to the distribution aspect of the TeleLWS_EG will be considered by our engineering specification as follows [3]:

1) The Presentation: The TeleLWS_EG will be presented as a 4-tier distributed system.

2) The interaction: The interaction aspect of TeleLWS_EG will be modeled according to a MVC architecture.

The embedded platform that represents the central element of the remote lab work activities will be accessible via the services of a SOA architecture. As shown in Fig. 1, this platform is composed of a remote embedded control devices combined with a 3D representation of an operative part of an automated system [4].

The second section of this paper gives an overview of the engineering specification of the TeleLWS_EG in UML4ODP.

The third section focuses on the node configuration model used to deploy the TeleLWS_EG.

The forth section concentrates on the description of the node structures of this configuration model in terms of Basic Engineering Objects (BeOs).
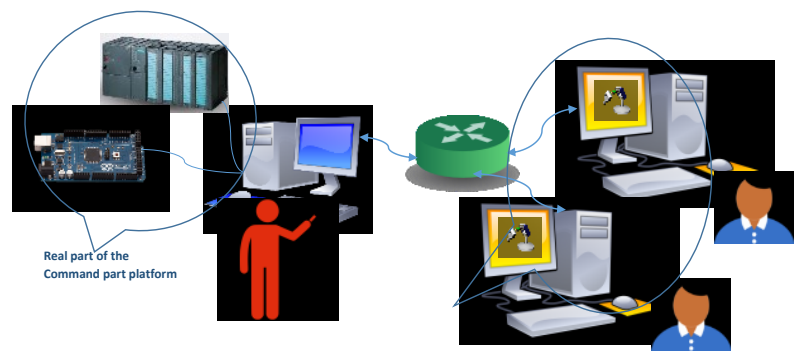

Fig. 1: The Labwork embedded platform. 


\section{AN OVERVIEW OF THE ENGINEERING SPECIFICATION OF THE TELELWS_EG IN UML4ODP}

The engineering language of the ODP model will enable us to express an engineering specification that will define the functions and the mechanisms required to support the execution and the distributed interaction between the engineering objects of the TeleLWS EG system.

From an engineering point of view, our system TeleLWS_EG will be specified as a distributed application composed of a set of basic engineering objects interacting via communication channels. The concepts of this language are: node (computer), kernel (operating system), capsule (address space) and cluster (modules linked to form an executable) and object engineering (program module).

All these concepts that express the engineering specification will be defined in a model, stereotyped as "Engineering_Spec" and written in the UML4ODP language [2].

Table 1 shows the schematic profile UML4ODP that summarizes the UML extensions for engineering language of the ODP_RM model.

To improve the clarity of the diagrams in this paper, the icons shown in this table will be used to represent the instances of the corresponding stereotypes.

TABLE I

ENGINEERING LANGUAGE ICONS

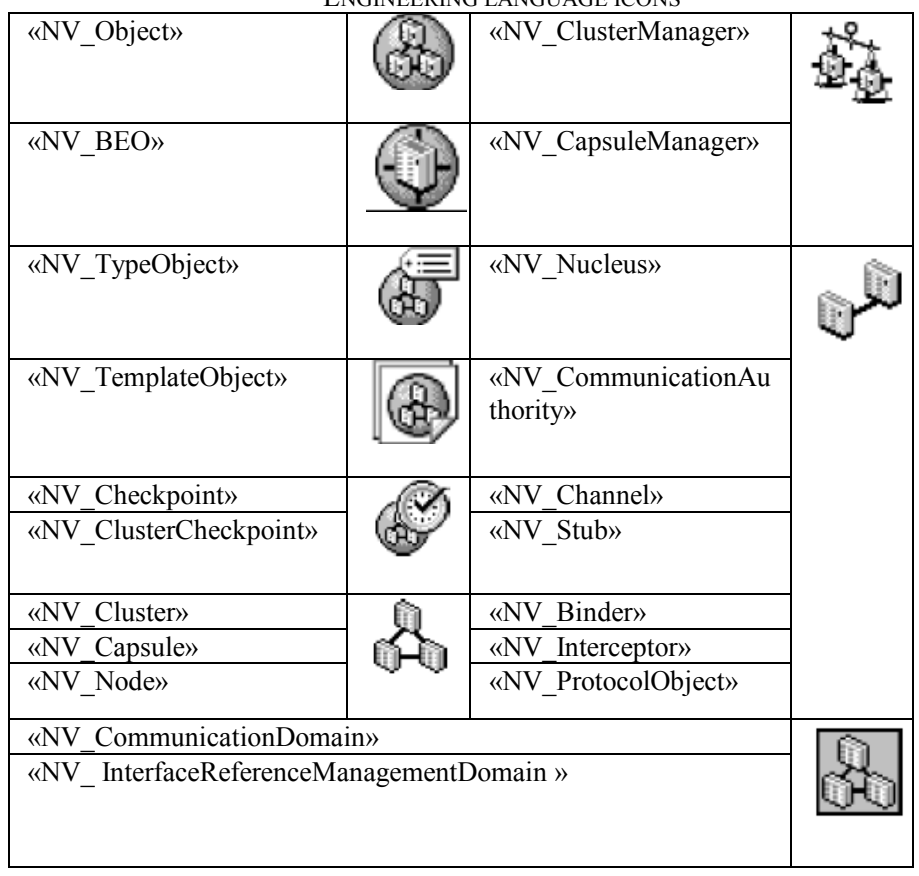

\section{NODE CONFIGURATION MODEL}

Like many standards our TeleLWS_EG distributed system for the development and execution of remote laboratory activities supports data persistence, transactions, security and business processing.
As shown in Fig. 2, the TeleLWS_EG distributed system consists of four nodes: ClientTier, InteractionTier, EnterpriseTier and MSRTier (MSR: Management of system resources).

The system users (learner, facilitator, author or administrator) can use a desktop PC or a mobile device, which serves as a ClientTier. An interaction request can be sent from the ClientTier to a web server which will be used as an InteractionTier. If necessary, the InteractionTier can send a processing request to an application server which is considered as an EnterpriseTier. Finally, the persistence and access to the resources is supported by the MSR_Server node that represents the MSRTier.

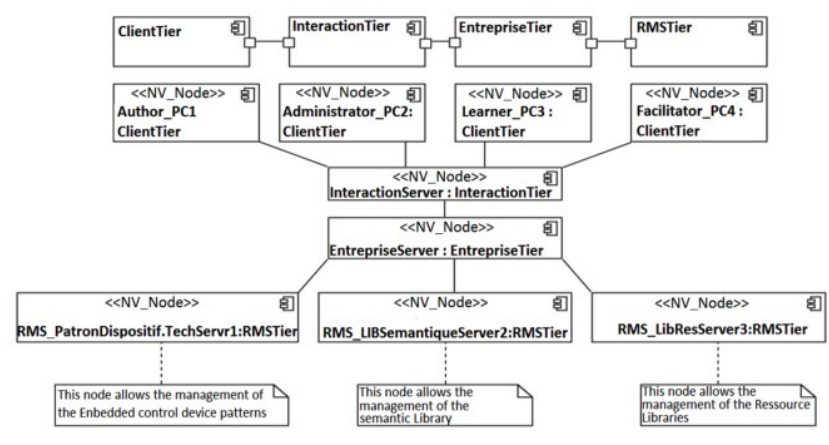

Fig. 2: Node configuration overview

\section{NODE STRUCTURES}

Each node consists of the node itself, nucleus, capsule(s), capsule manager(s), cluster(s), cluster manager(s), basic engineering objects (BeOs), engineering objects, stub(s), binder(s), protocol object(s), and interceptor(s).

"Fig. 3" shows an overview of the node configuration.

The computational objects that constitute the structure of the TeleLWS_EG system are called in the UML4ODP lingo BeOs. In the node configuration above, these $\mathrm{BeOs}$ are deployed as follows:

1) $\mathrm{BeOs}$ for graphical user interface allowing the access the system are deployed on the AssistantPC;

2) BeOs to supporting n-tier and MVC architectural style are deployed on the InteractionServer and the EnterpriseServer; and

3) $\mathrm{BeOs}$ for application specific computational objects are deployed on the EnterpriseServer and the MSR_Server.

"Fig. 4" shows the configuration of $\mathrm{BeOs}$ in the engineering architecture of the TeleLWS_EG system. BeOs are deployed on various nodes and within clusters in capsules. $\mathrm{BeOs}$ can have interactions between them by using channels.

$14^{\text {th }}$ LACCEI International Multi-Conference for Engineering, Education, and Technology: "Engineering Innovations for 


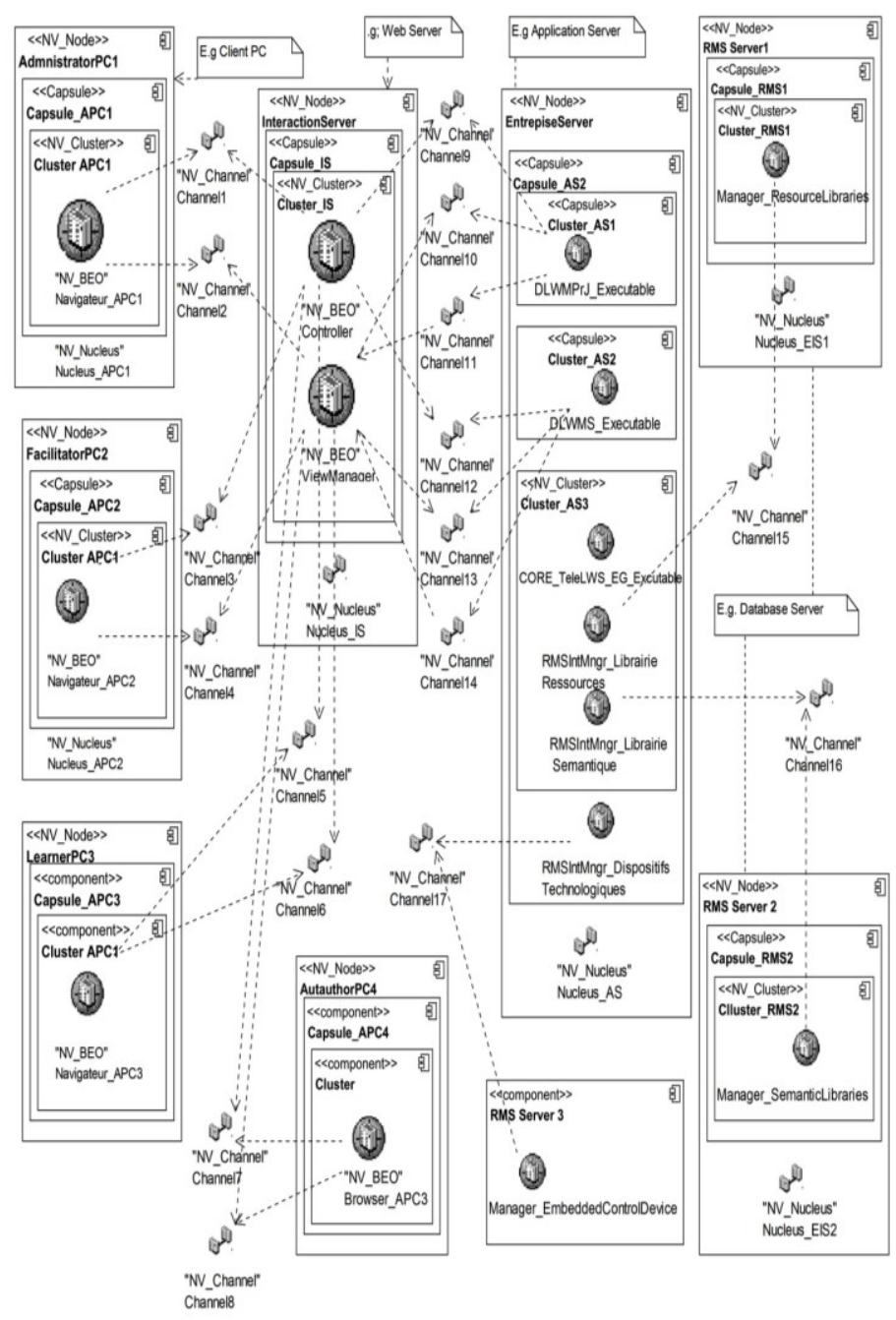

Fig. 3 BeOs configuration of the TeleLWS_EG (Engineering_Spec).

For instance, Browser_APC1 $\mathrm{BeO}$ on AssistantPC1 node communicates with Controller $\mathrm{BeO}$ on InteractionServer node via Channel1.

In the following clauses of this section we will present with more detail these $\mathrm{BeOs}$ by indicating the computational objects that they support.

\section{A. The BeOs of the ClientTier Node}

The $\mathrm{BeO}$ that is deployed on the "ClientTier» node is generally a GUI that allows the user to access to the TeleLWS_EG system via an interaction object.

This BeOS integrates usually a thin client application software which is extracted from the primary resource library (tools, documents, software locally installed, web browser, or applets Plugin) of the TeleLWS_EG system.

In the example shown by Fig. 5, this level consists of three nodes that are used by the main users (administrator, facilitator and learner) involved in the use and exploration of DLWMPrJ (Distance Lab Work Management Project).

Depending on the equipment installed at the client node, there is a wide variety of thin client software that can be used:

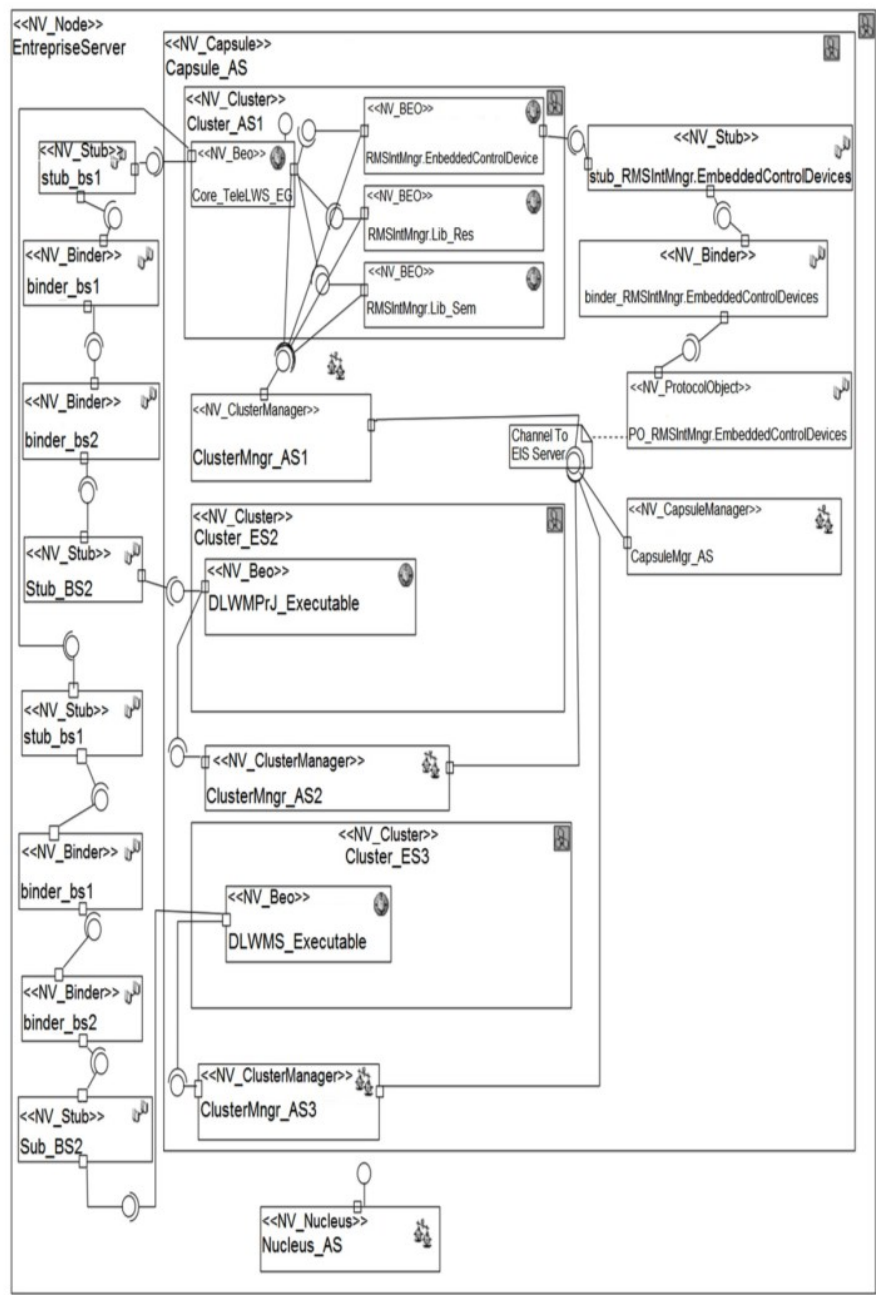

Fig. 4 Entreprise Internal Configuration Server

we can use a web browser with a standard desktop as we can use a wireless internet browser (WIB), supporting Wireless Markup language (WML) with a mobile device Like a Personal Digital Assistant (PDA).

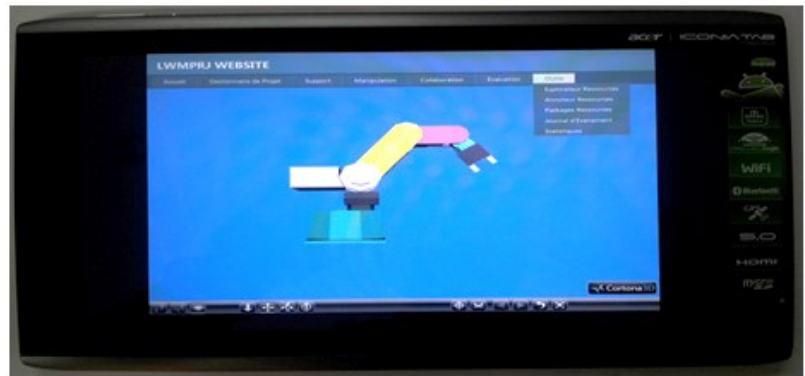

Fig. 5: Mobile Client node based on a two BeOs supporting a Wireless Internet Browser and a VRML Viewer.

$14^{\text {th }}$ LACCEI International Multi-Conference for Engineering, Education, and Technology: "Engineering Innovations for 
In a very particular context related to the teaching of the automated system the browser of the client node is requested to visualize the virtual mechanical part of the DLWMPrJ platform using a "3D viewer". This virtual mechanical part which is modeled in a 3D language (VRML) as a modular robot will be combined via a communication protocol with a real embedded control device during handling of the platform of TP@D.

\section{B. The BeOs of the InteractionServer Node}

The BeOs that represent the presentation objects and that support the MVC architectural model are deployed on two nodes: the InteractionServer and The EnterpriseServer nodes.

The system functions related to the presentation and to the interaction with the user and that must be provided by the TeleLWS_EG system are assigned to this type of BeOS. These $\mathrm{BeO}$ s allow the user to interact with the business objects in a secure and intuitive manner.

An interaction object is in fact an aggregation which is composed of three types of objects according to the MVC Model (Model-View-Controller): the controller object, the model object and the view object.

The controller is a control object which is always coupled to a view object. It handles user requires coming from the client workstations and orders a business or a model object to respond to the user's request appropriately.

For example, a learner who wants to look for a learning object representing a pattern of one of the components of the distance lab work platform can use a GUI provided by an interaction object to perform this research.

The interaction object submits this order to a business object, which checks the platform's components pattern libraries that are available via a manager object that performs the necessary functions for accessing and sending.

Once the user request was processed, the system can provide various views. The current examples are:

1) A GUI displaying a $3 \mathrm{D}$ learning Robot to be manipulated by the learner as shown by Fig. 6 ;

2) A GUI showing the virtual control device that will be programmed by the learner.

\section{LWMPR WEBSTIE}

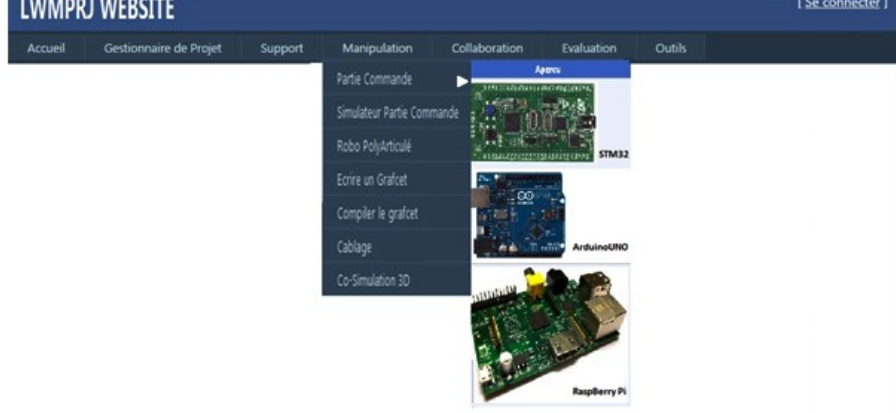

Fig. 6: The view of the BeO supporting the DLWMPrJ interactive Environment delivered by the InteractionServer Node.

\section{The BeOs of the EnterpriseTier Node}

The BeOs corresponding to the business computational objects and the interfaceGRS's manager objects are deployed on the EnterpriseServer node.

1) The "EnterpriseTier" includes Enterprise components that represent the business logic of the TeleLWS_EG system.

2) The system calls to these objects are performed by the ClientTier via the InteractionTier (WebTier).

3) If necessary the EnterpriseTier interchanges with the recording layer (RMS: Resources Management System) to access to the resources invoked by the said calls. These business objects are responsible the production system of the TeleLWS_EG's aggregation resources that are organized in four levels of a cascaded architecture [5] as shown in Fig. 7. These objects are particularly global: The first level of this production cascade which is represented by the basic system "TeleLWS_EG Core" allows us to produce the objects of the second level. These ones are really a set of distance labwork platforms and they are technically known as DLWMS (Distance Labwork Management System). The DLWMS are used to generate the objects of the third level of our cascade that are called DLWMPrJs (Distance Labwork Management Project). The DLWMPrJ which is an application software used as an environment to remotely practice Lab works on embedded system. It is used to produce a set of aggregated products that will constitute the fourth level of the cascade. These products are the results of the practice activities performed by the different actors concerned by the execution of the DLWMPrJ [6]. These products are assembled in a set of portfolios (portfolio) or DLWMPrDs (distance Labwork Management Product).

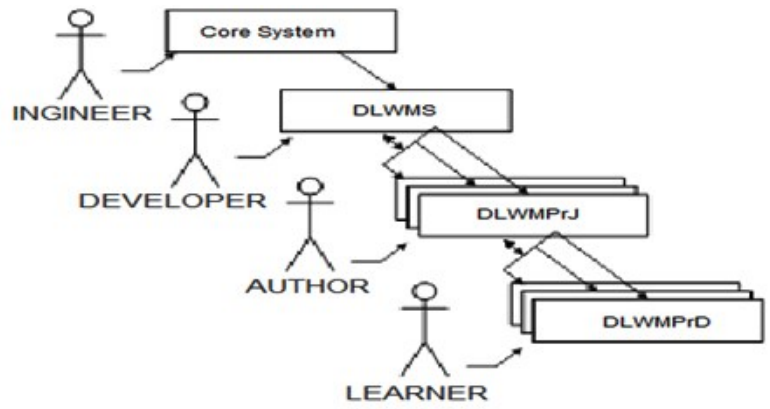

Fig. 7: The four levels of the TeleLWS_EG's cascaded architecture.

\section{The BeOs of the RMS_Server Nodes}

The BeOs corresponding to the computational objects of the library managers are deployed on the RMS_Server nodes.

The RMSTier level (Resource Management System Tier) allows the enterprise objects to be independent from the library and the physical resources of the system.

This level of the network architecture includes three nodes:

$14^{\text {th }}$ LACCEI International Multi-Conference for Engineering, Education, and Technology: "Engineering Innovations for 
1) The first node is used to deploy the managers of the different types of the resource libraries that contain the entire information system of the TeleLWS_EG, except the managers of the embedded system control devices that are deployed on another node. These libraries include different types of resource aggregations [5] [6]: 1) the primary resource libraries include documents and tools (D, T), Users (U) and operations (O). A simple text editor that lets create a control code to be executed by the control device can be among the tools of these libraries; 2) the secondary resource libraries comprises the interfaced resources (the interaction objects) resulting from the combination of a control component or a HMI with a primary resource or from the aggregation of primary or interfaced resources; The four types of HMI (Assembly O.P Diagram HMI, Datalink Map Connection HMI, Programming HMI and Cosimulation HMI modelling) that we have developed and that constitute the modelling and simulating system of the $3 \mathrm{D} \mathrm{Web}$ Based robots that are coupled with control devices to teach embedded systems, can be considered as secondary resources; 3 ) the tertiary resource libraries grouping the most important enterprise objects of our TeleLWS_EG system: Core system, DLWMS, DLWMPrJ and DLWMPrD.

2) A second node for managing the semantics library of the TeleLWS_EG system. This node contains several semantic repository managers.

The mainly managers that are deployed on that node are those of ontologies [7] written in SOCOM (Software Component Metadata).

We have adopted this semantic representation to construct the semantic repositories that describe the system resources and their administrative and technical context of use. One of the specific ontology that was used has been defined as spatiotemporal patterns [2] which combines the concepts of SOCOM [7] and MADS [8] formalism to describe a 3D Semantic Data Library that produce descriptions of 3D robots for distance Labwork.

3) A third node for the management of the control devices embedded systems: due to many reasons related to the genericity of the distance labwork scenarios and to the specificities that characterize access to the control component of the embedded automated systems, we have chosen to deploy their manager on an independent node.

In fact, the manager of these devices is a very specific tool that ensures mainly the functions of referencing, research and access to these control components.

The Control devices of the embedded automated systems, are declared in the Labwork scenarios of our Generic environments (PLC, microcontroller etc.) as Universal Resource Locators (URLs) to the desired devices like any other object (document, script, etc.) on the Web [9].

To ensure the genericity of these scenarios, it is necessary that these URLs localize the control devices and their expected functionalities and not the address of the labwork platform server because in this case the scenario would be specific to a single labwork platform in the world.

It is possible to achieve this objective, through the use of an embryonic ontology that we have built to describe the components of a real control device associated with their functionalities.

This ontology that we have built is an extension of the metadata specification SOCOM defined in [5] for the referencing of the software components.

Once a new type of a control device or an extension of an existing type is physically installed, the administrator of the labwork platform uses Control device Interface Management Tool to declare it as the device access server by creating a new pattern or, a pattern derived from an existing pattern (to limit the declaration of additional components and functionalities) and thus make it available to users.

Once the pattern is created, the administrator must declare (by using the manager) the links between the functionalities described by the pattern and the physical hardware components (for example, the functionality "Load program" of the component "microcontroller" is associated with the URL "http://41.229.255.197:8080/FournisseurService/NIcRio9025/ UC/Load" of the control unit of the device "microcontroller National Instrument cRIO-9022" [10]).

The SOA (Service Oriented Architecture) architecture based on the JNI (Java Naming Interface) technology shown in Fig. 8 is proposed for the implementation and integration of distributed embedded control devices whose components and services are accessible via the device access server. We will adopt this architecture to describe a thin environment (the device access server) for exploration and use of services that represent fast connectable embedded control devices [11].

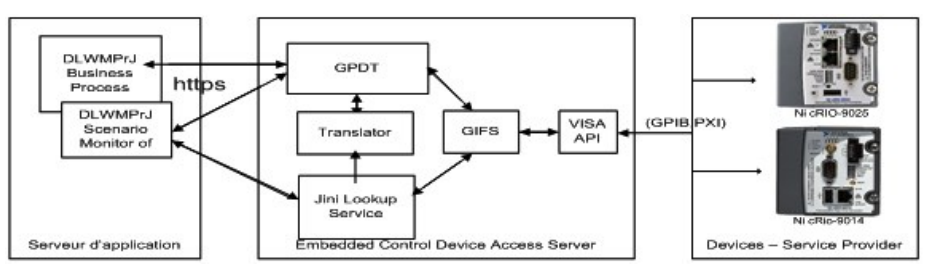

Fig. 8: Embedded Control Device Access Server based on Jini Technology.

\section{CONCLUSION}

This paper has discussed the specification of the TeleLWS_EG's system from an ODP engineering point of view. In the ongoing work we will describe the implementation of the TeleLWS_EG's system from an ODP technological point of view. This point of view ODP will allow us to describe the realization of the TeleLWS_EG system in terms of technological object configuration that represents hardware, software and network components of the system. We believe

$14^{\text {th }}$ LACCEI International Multi-Conference for Engineering, Education, and Technology: "Engineering Innovations for 
that this paper is a valuable contribution for the IEEE-SA P1876 ${ }^{\mathrm{TM}}$ Working Group.

\section{REFERENCES}

[1] H. Saliah-Hassane, A. Kourri, I. De la Tejal, "Building a repository for online laboratory learning scenarios", Frontiers in Education Conference, 36th Annual Volume Issue 27-31, pp.19 - 22, October 2006.

[2] Information technology - Open distributed processing - Use of UML for ODP system specifications, ITU-T, October 2014. http://www.lcc.uma.es/ av/download/UML4ODP_IS_V2.pdf

[3] M. Mhamdi, H. Saliah-Hassane and R. Braham, "Expressed in UML4ODP: an Engineering Specification of a Distributed Environment for Teaching Embedded Systems", ICEER2013 (International Conference on Engeneering and Education and Research), Marrakech,Maroco, Juillet, 2013.

[4] M.Mhamdi, R.Braham, M.Moalla, H.Saliah-Hassane, "A Virtual Verification and Execution of Grafcet Using VRML", Special Volume:ENGINEERING EDUCATION AND RESEARCH, pp 381-389, 2004.

[5] A. Masmoudi, G. Paquette, R. Champagne, "Metadata-Based software components repository's reuse", $3^{\text {rd }}$ Annual Scientific Conference LORNET Research Network, Montreal, Quebec, 2006.

[6] G. Paquette, F. Magnan, "An executable model for virtual campus environments", Handbook on Information Technologies for Education, eds A. sberger, H. Heimo, K. Pawlowski, J. Martin, Springer. pp. 363403, 2008

[7] A. Masmoudi, et al. "Développement d'un scénario de laboratoire en ligne par une agrégation de composants logiciels basée sur les ontologies", The $3^{\text {rd }}$ International Symposium on Distance Education, Tunisia, 2006.

[8] A.Vakaloudis, B. Theodoulidis, "Formalizing interactive behavior in 3Dspatiotemporal worlds", the working conference on advanced visual interfaces, Palermo, Italy, 2000.

[9] A. Lelevé, H. Benmohamed, P. Prévôt, "Mise à distance de travaux pratiques en automatique", Revue Skholê: cahiers de la recherche et du développement, Vol. 14, pp 87-99, 2008.

[10] User manual and specifications, Intelligent real-time embedded controller for CompactRIO, NI. http://www.ni.com/pdf/manuals /375102g.pdf

[11] F. Bouchaib, "Granularité et agrégation des interfaces graphiques de laboratoires en ligne distribués", Ecole de technologie de l'information, Université du Québec, 2005.

$14^{\text {th }}$ LACCEI International Multi-Conference for Engineering, Education, and Technology: "Engineering Innovations for Global Sustainability”, 20-22 July 2016, San José, Costa Rica. 